\title{
Delivering integrated child development care in Pakistan: protocol for a clustered randomised trial
}

\author{
Muhammad Amir Khan, DHA, MPH, PhD, FFPH ${ }^{1}$, Syeda Somyyah Owais, MSPH ${ }^{2 *}$, \\ Claire Blacklock, MRCP, MRCGP, DTM\&H, PGDip ${ }^{3}$, Shirin Anil, MSc, MBBS ${ }^{4}$, \\ Sehrish Ishaq, MSPH, PGD Community Health and Nutrition ${ }^{5}$, Shazia Maqbool, MD Paeds, FRCPCH ${ }^{6}$, \\ Haroon Jehangir Khan, BSc, MA HMPP, MPH, FRIPH ${ }^{7}$, \\ Fareed A Minhas, MSc, DIP (Psych), MCPS, MRCPsych ${ }^{8}$, John Walley, MComH, FFPH, MRCGP9
}

${ }^{1}$ Chief Coordinating Professional, Association for Social Development, Islamabad, Pakistan; ${ }^{2}$ Project Manager, Association for Social Development, Islamabad, Pakistan; ${ }^{3}$ Lecturer in International Public Health, Nuffield Centre for International Health and Development, Leeds Institute of Health Sciences, University of Leeds, Leeds, UK; ${ }^{4}$ Project Coordinator and Consultant, Association for Social Development, Islamabad, Pakistan; ${ }^{5}$ Project Coordinator, Association for Social Development, Islamabad, Pakistan; ' $P$ rofessor of Developmental Paediatrics, Institute of Child Health and The Children's Hospital, Lahore, Pakistan; ${ }^{7}$ Focal Person (Non-Communicable Diseases), Directorate General of Health Services Punjab, Lahore, Pakistan; ${ }^{8} \mathrm{Head}$, Institute of Psychiatry, Rawalpindi, Pakistan; ${ }^{9}$ Professor of International Public Health, Nuffield Centre for International Health and Development, Leeds Institute of Health Sciences, University of Leeds, Leeds, UK

*For correspondence: gcc@asd. com.pk

Competing interests: The authors declare that no competing interests exist.

Received: 26 August 2016 Accepted: 22 September 2016 Published: 09 January 2017

(c) This article is Open Access: CC BY license (https:// creativecommons.org/licenses/ by/4.0/)

Author Keywords: primary care, primary health care, general practice, Pakistan, child development, nutrition, depression

Copyright (C) The Authors 2017; DOI:10.3399/

bjgpopen17X100677

\section{Abstract}

Background: Early childhood developmental delay is associated with significant disadvantage in adult life. In Pakistan, high prevalence of developmental delay is associated with poverty, undernutrition, and maternal depression.

Aim: To assess the effectiveness of an early child development counselling intervention delivered at private GP clinics, in poor urban communities.

Design \& setting: A clustered randomised trial in Pakistan.

Method: The intervention was developed following a period of formative research, and in consultation with local experts. A total of 2112 mother-child pairs will be recruited at 32 clinics, from within the locality (cluster); 16 clinics per arm. A primary care counselling intervention (promoting child development, nutrition, and maternal mental health) will be delivered at 6 weeks, 3,6 , and 9 months of the child's age. Monitoring, assessment, and treatment will also be performed at quarterly visits in intervention clinics. Primary outcome is the developmental delay at 12 months (ASQ-3 scores). Secondary outcomes are stunting rate, and maternal depression (PHQ-9 score). In addition, a process evaluation and costing study will be conducted.

Discussion: This trial will be the first to assess an early child development intervention, delivered in private GP clinics for poor urban communities in Pakistan. If found to be effective, this publicprivate model may offer a more sustainable, and feasible option for populations in poor urban settings, where private GP clinics are the most accessible provider of primary health care. There is scope for scale-up at provincial level, should the intervention be effective. 
Trial registration: The trial has been registered with the Current Controlled Trials ISRCTN48032200.

\section{How this fits in}

In Pakistan, the current mother and child care at private GP clinics does not cover the child brain development and mother mental health components, and nutrition counselling is also suboptimal, mainly due to lack of context-sensitive tools and inadequate staff ability and engagement. The referral linkages with public hospitals are also inadequate for mothers to access an expert consultation for their child development challenges. The intervention offers a set of primary care guidelines and tools for sustainable district health stewardship of private GP clinics to promote optimal early development of urban poor young children (that is, first year of life), through an integrated package of mother and child care. These women currently fail to access early child development care because of overloaded public hospitals, the inability of unregulated private clinics, and lack of affordability. This 'action' will solve the problem by making early child development care package available at selected private GP clinics. The care package will have three main components: nutrition, child development, and maternal mental health.

\section{Introduction}

Childhood developmental delay is associated with poor subsequent educational attainment, and lower income during adult life, contributing to a cycle of poverty. It is a significant public health concern in many low- and middle-income countries, including Pakistan. ${ }^{1}$

Prevalence of developmental delay in Pakistan has been estimated at around $15 \%$, ${ }^{2}$ even exceeding $30 \%$ among children from poorer families. ${ }^{3}$ Developmental delay is strongly linked to undernutrition, ${ }^{4-6}$ and maternal depression. ${ }^{7}$ Indeed, inadequate stimulation in early childhood, and maternal emotional characteristics, are associated with poorer cognitive development. ${ }^{4}$ In one Pakistani study more than one-quarter of new mothers had depression. ${ }^{8}$

There is evidence from low resource settings that programmes to improve infant stimulation and enhanced parenting skills have a beneficial effect on development. ${ }^{9-11}$ In the Caribbean, a parenting intervention integrated into routine primary care was effective in improving cognitive development. ${ }^{12}$ In Pakistan, a development and nutrition intervention delivered by community 'lady health workers' in Sindh province, had a significant positive effect on development outcomes. ${ }^{13}$

In poor urban areas of Pakistan, public primary care services are scant, and referral hospitals difficult to access due to distance, expense, and high demand. Primary care is therefore commonly provided by private GP clinics and small community hospitals. These are unregulated, unsupported, and vary in both quality and cost of services. Existing maternal and child health services do not adequately meet the early child development and maternal mental health needs of the population, and nutritional counselling is lacking. ${ }^{14}$ This trial has been designed to investigate the effectiveness and feasibility of integrating an early child development intervention into existing private GP clinic services for poor urban communities, and the aim will be to assess the effectiveness of a primary care intervention in reducing early developmental delay, in poor urban communities of Pakistan, using public-private partnership.

\section{Method}

\section{Study design}

Pragmatic, parallel arm, clustered randomised controlled trial with two arms (intervention and control) will be conducted to compare an early child development intervention delivered in private urban GP clinics in Pakistan, with usual routine care. Thirty-two clusters will be randomised using a 1:1 ratio, to intervention or control Figure 1. 


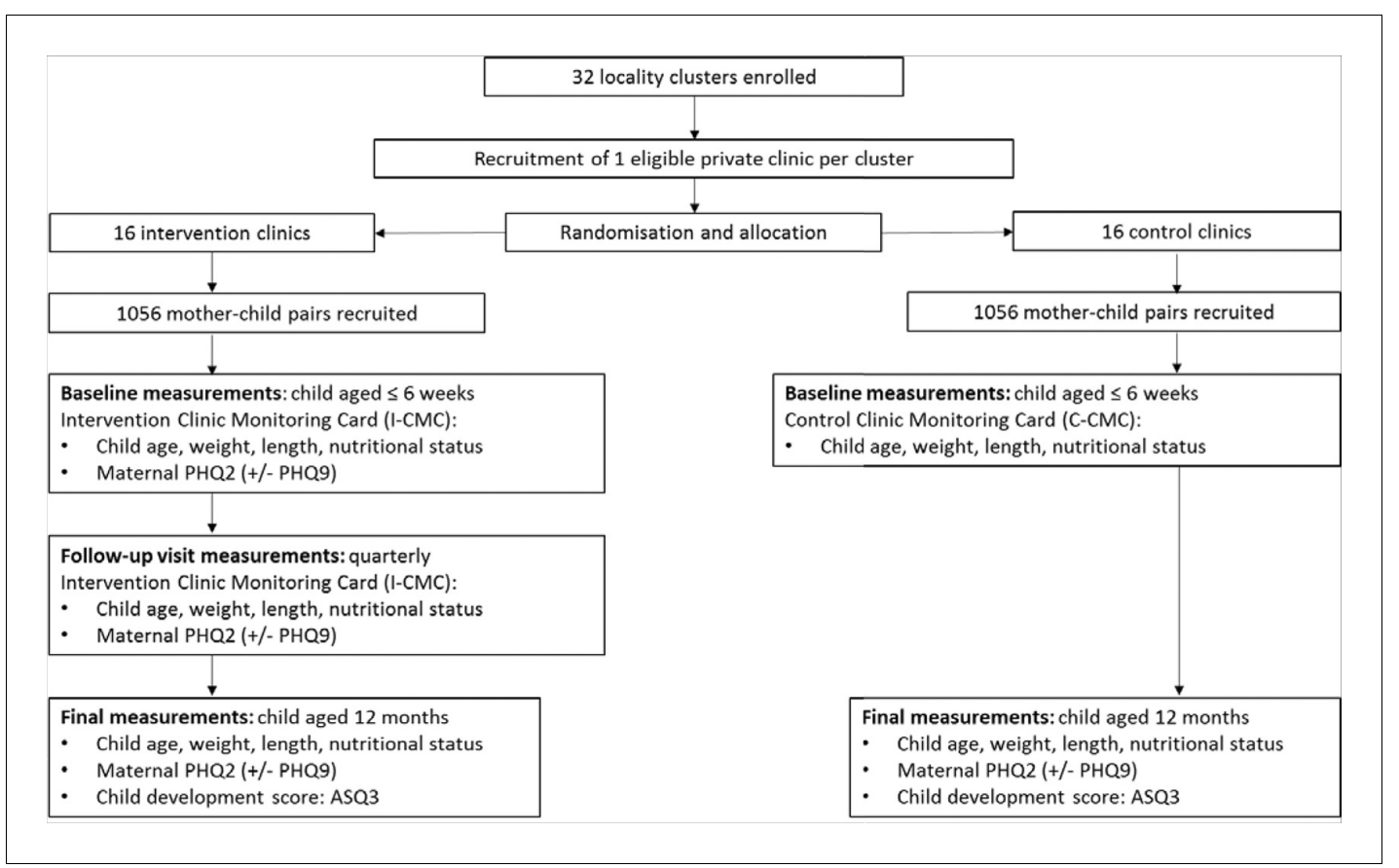

Figure 1. Trial flow diagram

\section{Setting}

The districts of Lahore and Rawalpindi are study sites, following consultation with the Directorate General Health Services (DGHS), Children's Hospital, and the Institute of Psychiatry. Lahore and Rawalpindi have large urban poor communities, and an extensive existing network of private GP clinics and small private hospitals. Average household size is 7.2 and 6.5 in Lahore and Rawalpindi respectively. Female literacy is around $60 \%$ in both districts. Electricity is available to more than $90 \%$ of households, but piped water to only $75 \%$ and $41 \%$ in Lahore and Rawalpindi respectively (and gas for cooking, $63 \%$ and $53 \%)^{15,16}$

\section{Clusters}

Each trial cluster will represent one priority poor urban locality settlement, with limited access to a public hospital, and an average population of 50000.

\section{Trial clinics}

Within each cluster, one study clinic will be selected from available private GP providers, with preference for longer established clinics, and existing providers of maternal and child care. Private clinics typically comprise one doctor (GP), and employ at least one clinic assistant. Clinic assistants are usually males from the local area, with between 10-12 years of schooling, but no formal paramedic training. ${ }^{14}$ They are trained on-the-job by the private doctor, and support the private doctor by dispensing medication and performing other tasks, as necessary.

\section{Recruitment of mother-child pairs}

Eligible mother child pairs will be recruited at participating private clinics by clinic assistants trained in trial recruitment and consent procedures. Recruitment will continue up to 6 weeks of child's age, to allow for cultural restrictions during first 40 days postpartum.

\section{Inclusion criteria}

- Mother-child pair

- Child $\leq 6$ weeks 


\section{Exclusion criteria}

- Prematurity (<36 weeks gestation), history of congenital abnormality, delayed cry at birth, seizures, cretinism, or low birth weight $(<2500 \mathrm{~g})$

- Mother intends to move out of area during study period

- Child not accompanied by mother (for example, accompanied by another relative)

\section{Intervention}

The intervention was developed following a period of formative research. ${ }^{14}$ In brief, this focused on understanding the context within private GP clinics, and experiences and preferences of mothers. Feasibility and acceptability of proposed intervention components were explored, and findings used to inform intervention development. The outline is described in Box 1.

Box 1. Early child development care in private GP clinics

Control clinics

Mother-child care Usual care

Intervention clinics

- Standard counselling session on childhood nutrition, development, and maternal mental health, using pictorial flipbook.

- Monitoring and screening of child growth and maternal mental health.

- Assessment and treatment (including referral to specialist) of childhood nutrition, development, or maternal depression.

- Follow-up of mother-child pairs in clinic at 3,6 and 9 months (including SMS or telephone reminder, if required)

\begin{tabular}{lcc}
\hline Low dose vitamin A & Yes & Yes \\
\hline Community advocates & Yes & Yes \\
\hline $\begin{array}{l}\text { Branding of clinic by } \\
\text { Directorate General }\end{array}$ & Yes & Yes \\
Health Services & &
\end{tabular}

Services

Training of private Control clinic staff will receive basic training only, focused on doctors (GPs) and clinic the correct use of study recording forms, with a general assistants overview of the importance of childhood development and maternal health; that is, they will not receive any specific training on intervention activities.
Clinic assistants will be trained by project field coordinator (under supervision of project manager and specialist doctor) using the study clinical assistant training protocol (CATP), which includes:

- how to conduct a standardised counselling session using the flipbook;

- how to administer the PHQ-2; and

- how to measure and record child length and weight.

Private GPs will be trained by an experienced specialist according to the study private doctor training protocol (PDTP), which includes:

- clinical management of children with malnutrition and developmental delay in the private clinic setting;

- how to use PHQ-9 for the diagnosis of maternal depression; and

- how to assess the mother-child pair for a specialist referral, when required, to the appropriate public tertiary care facility.

The training protocols, both CATP and PDTP, are developed by a group of local experts and specialists, adapted from international best-practice guidelines and standards. ${ }^{16,17}$

Training will last approximately 2 hours, and will include a mixture of explanation by the project field coordinator, and role-play exercises by participants. 
Maternal counselling (core intervention)

A quarterly maternal educational counselling session with the clinic assistant at the private GP clinic will promote early child development, nutrition, and maternal mental health. Counselling content is developed from existing guides, in consultation with technical experts, ${ }^{17,18}$ supported by a pictorial flipbook.

Counselling will encourage mothers to engage in activities, appropriate for the expected stage of child development. It will also focus on nutrition and maternal mental health messages. Content was adapted for the context in Pakistan through a process of expert consultation. Trained and experienced artists were engaged to illustrate key activities and messages. The pictorial flipbook is twosided: one side with pictures of activities to be shown to the mother; and the other with prompts for the clinic assistant. Each counselling session comprises 4-5 pages of the flipbook, and takes no more than 10 minutes to deliver.

Mother-child pairs will be invited to return for follow-up every 3 months, to receive the next counselling session; that is, at 6 weeks, then at 3, 6, and 9 months of the child's life.

\section{Assessment and treatment of child and mother}

At each visit, the clinic assistant will monitor the growth of the child, and screen for maternal depression (PHQ-2 score). ${ }^{19,20}$ Referral will be made to the private GP for further assessment where indicated.

Where indicated, the private GP will assess the child for malnutrition and developmental delay, and the mother for depression using the PHQ-9 score, ${ }^{19,20}$ and manage any concerns, including referral to a specialist if necessary.

\section{Ancillary intervention components}

Maternal counselling, and assessment and treatment, will be supported by:

- Training of private GPs and clinic assistants

- SMS reminders to mothers for follow-up visits

A logic model and theory of change for the intervention is described in Box 2, drawing on the refined Theoretical Domains Framework. ${ }^{21}$

\section{Public-private partnership}

Both intervention and control clinics will be endorsed by the DGHS to advertise the availability of early childhood development care to the community.

\section{Community advocates}

Participation of mother-child pairs in the study will be encouraged by institution-based members of the community (such as the barber shop or grocery store) advocating for clinics, including distributing study leaflets to interested clients.

\section{Control clinics}

Control clinics will continue usual mother-child care. Control clinic staff will receive basic training only, focused on the correct use of study reporting forms, and a general overview of childhood development and maternal health, that is no training in counselling, or in assessment and treatment of mother-child pairs, or provision of flipbook.

Both intervention and control clinics will receive standardised calibrated equipment (digital scales, and infantometer), to ensure quality of study data. Clinic assistants will be instructed to maintain records in kilograms and centimeters, to ensure uniformity in measurements. Intervention and control clinics will receive a small monthly reimbursement, for participation in research activities.

\section{Outcomes}

Primary outcome

Suspected developmental delay on screening: ASQ-3 score at 12 months

The Ages and Stages Questionnaire $3^{\text {rd }}$ Edition (ASQ-3) is a childhood developmental screening tool. ${ }^{22}$ By assessing across five developmental domains, it identifies children in primary care who are 
eligible for further assessment. It is intended that the parent completes the questionnaire, after observing the child at home. However, it can be adapted, for example a parent can be assisted if literacy is poor. Translation and cultural adaptation of the questionnaire is also recommended. ${ }^{23-}$ 25 Internal and external validity trials of ASQ in a range of populations, cultures, and settings report mixed findings. ${ }^{24,26-30}$ The ASQ-3 questionnaire for children aged 12 months is reported to have a sensitivity of $90 \%$ and specificity of $87.5 \%$, when compared to equivalent cutoffs using the Battelle Developmental Inventory-II. ${ }^{31}$ A trial of Hindi ASQ-3 questionnaires in a South Asian population of high and low risk children, found an overall sensitivity of $83.3 \%$ and specificity of $75.4 \%$. $^{32}$ The 'home procedure' was feasible in India when delivered by non-psychologists, under instruction and supervision. Inter-observer reliability was good. Overall internal validity of the questionnaires was also good, but varied at subscale level. ${ }^{25}$

The ASQ-3 questionnaire has been translated into the Urdu language and adapted to local context for this study. ASQ-3 assessors will have a bachelor's degree in psychology, and receive training and supervision. Assessment will be conducted at 12 months of age, in private GP clinics. The assessor will record: 1) mother's responses to the ASQ-3 questionnaire, based on recall and any self-initiated observations; and 2) the assessor's own on-site observations of specific activities during the assessment session, making use of appropriate toys and props. Mother and assessor scores will be analysed separately, and inter-rater agreement assessed. Final ASO-3 outcome score will be a composite score of mother response and on-site observations.

\section{Secondary outcomes}

\section{Childhood nutrition status: weight ( $\mathrm{kg}$, percentile), length ( $\mathrm{cm}$, percentile) at} 12 months

Each child will be measured for weight and length by the clinic assistant. Measurements will be plotted on a growth chart, and percentiles calculated. The prevalence of underweight and stunting will be calculated.

\section{Maternal depression: PHO-9 score at 12 months}

Maternal depression will be assessed at 12 months by administering the PHQ-9 questionnaire, already available in the Urdu language. ${ }^{33}$ PHQ-2 and PHQ-9 are validated screening tools for depression. ${ }^{19,20}$

\section{Data management}

\section{Participant registration and monitoring data}

Sociodemographic information will be recorded at participant enrollment. Ongoing clinical data will be collected by the clinic assistant. Completeness of data collection will be checked by a research officer, who will update trial records, kept securely at the research office. Anonymised electronic data will be entered into SPSS Version 20.0, stored on a password protected computer.

\section{2-month outcome data}

All mother-child pairs will be invited to a 12-month final outcome assessment visit. Hard copies of outcome assessment forms will be kept securely, and anonymised electronic data inputted into SPSS (version 20.0) for analysis.

Analysis

Both individual and cluster level analysis will be conducted according to intention to treat in SPSS version 20.0 and R version 3.2.3 respectively. Intervention and control arms will be compared at baseline to ensure equal distribution of potential confounders like maternal education, the purpose of randomisation. Scores will be calculated for the five domains of developmental delay namely gross motor, fine motor, problem solving, and personal-social using ASQ3. The two arms will be compared for these five domains of development delay at 12 months by independent sample $t$-test adjusting for clustered data, as used by other researchers, ${ }^{34}$ by applying t.test.cluster in Hmisc package in R. Stunting will be measured at end line using WHO Anthro SPSS Macros. The two arms will be compared with respect to stunting by $\chi^{2}$ test adjusted for clustering ${ }^{35}$ by applying donner function in aod package in R. ${ }^{36}$ Mother's mental health condition will be compared across both arms as 

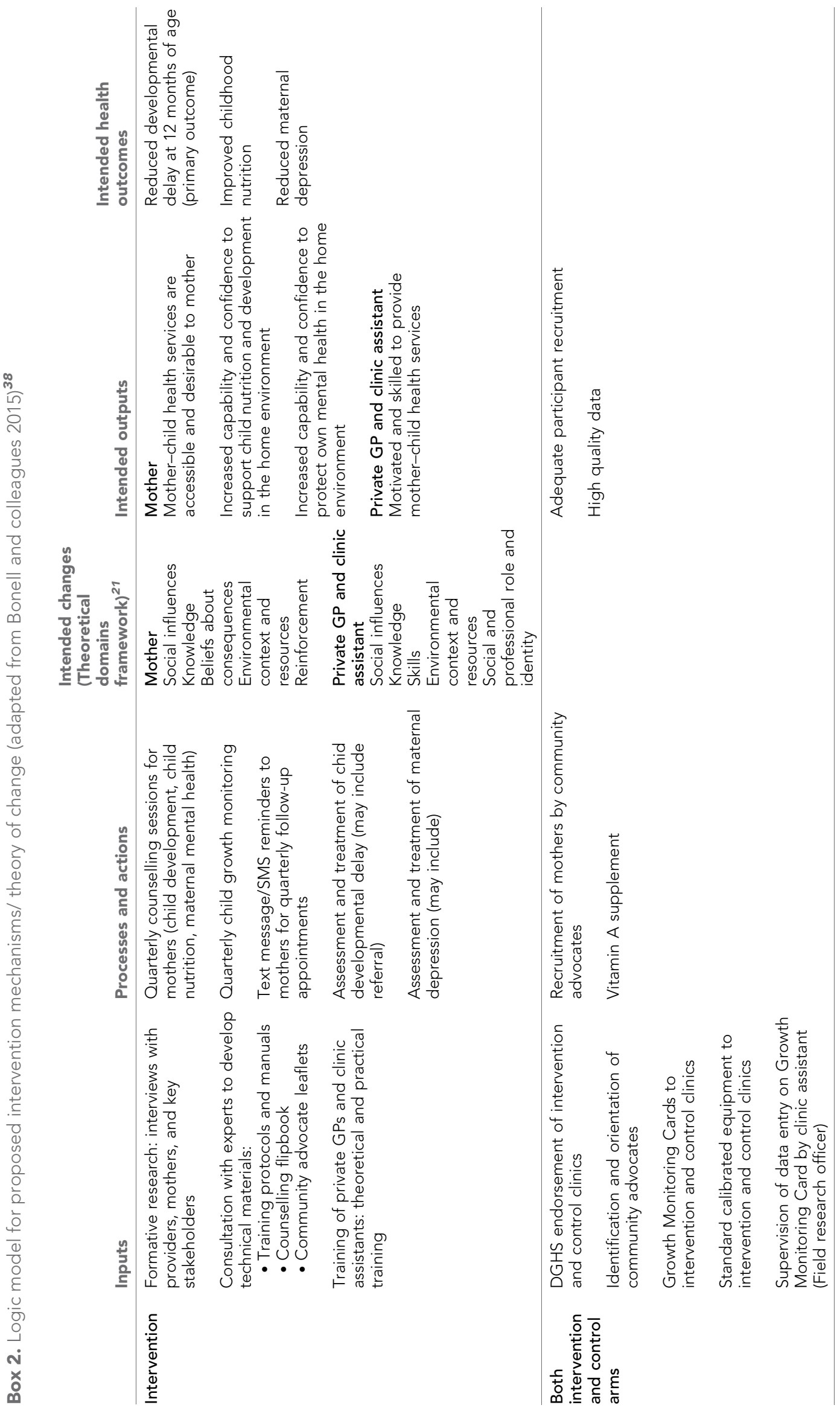
end line using PHQ-9 scores $t$-test adjusting for clustered data. ${ }^{34} \mathrm{~A} P$-value $<0.05$ will be considered significant. Generalised estimating equation (GEE) and generalised linear mixed models (GLMM) will be applied using $\mathrm{R}$ packages gee and glmmML respectively in case of residual confounding. ${ }^{37,38}$

Sample size calculation

At least 1056 mother-child pairs will be required (in 16 clusters: 66 mother child pairs per cluster) in each of the intervention and control arms (a total of 2112 mother-child pairs in a total of 32 clusters) to detect a difference of $20 \%$ in development delay (assuming $33 \%$ suspected development delay $^{39}$ at baseline) at $80 \%$ power, $5 \%$ level of significance and an intra-class correlation coefficient of 0.15 for maternal education, ${ }^{13}$ allowing for $10 \%$ loss to follow-up in each arm.

\section{Randomisation}

Simple randomisation will be performed by drawing from a hat, by a member of the host research organisation not involved in the study. Stratification by districts (Rawalpindi and Lahore) has not been done as both share similar characteristics being major cities in the province of Punjab. Nevertheless clinic size, if not equally distributed between both arms by randomisation, will be taken care of in modeling (GEE and GLMM as mentioned above).

\section{Blinding}

Due to the nature of the intervention, participants will not be blinded. ASQ-3 assessors will be blinded to allocation of participants. Members of the research team undertaking 12-month maternal PHO-9 assessments will not be blinded.

\section{Ethics and dissemination}

Verbal consent will be obtained from community leaders. Written consent will be taken from the clinic staff, and verbal consent from participating mothers. Mothers can withdraw consent at any time. Clinical referrals will be made to local specialist services where appropriate.

The trial results will be published in open access peer-reviewed journals. Results will be communicated to key government and non-government stakeholders at national level, with a view to scale-up if the intervention is effective, feasible and acceptable.

The SPIRIT checklist has informed the preparation of this protocol. ${ }^{40}$

\section{Process evaluation}

A process evaluation will accompany the trial, following MRC Guidance. ${ }^{41}$ Mixed methods will be used to explore intervention implementation, mechanisms of impact, and context. Quantitative data will be extracted from study records, and descriptive analyses presented. Qualitative data will be gathered from in-depth interviews. Purposive sampling will be used, for maximal variation in views and experience. Interviews will be audiorecorded and transcribed in Urdu. Framework Approach will be used in analysis. ${ }^{42}$

\section{Economic evaluation}

Data will be collected on costs incurred by the intervention to inform incremental cost-effectiveness ratio (ICER) analysis of the health services cost. The study will account for both provider and client perspectives, and will consider recurrent costs incurred should the intervention be implemented without study resources. The marginal costs of the intervention, to both public and private providers will be estimated, as well as costs to participating mothers. An economic survey of participating mothers will be conducted at 12 months to determine costs of care.

\section{Discussion}

\section{Summary}

A clustered randomised controlled trial will assess the effectiveness of an early child development counselling intervention delivered at private GP clinics, in poor urban communities of Pakistan. 
Primary outcome is the developmental delay at 12 months (ASQ-3 scores). Secondary outcomes are stunting rate, and maternal depression (PHQ-9 score).

\section{Strengths and limitations}

The intervention was developed in consultation with local experts, following a period of formative research. It will be implemented in the existing private GP clinic setting, in the context of a publicprivate partnership. Anticipated challenges of the proposed research include:

- Follow-up appointments are not usual practice in the existing primary care context in Pakistan. Non-adoption by providers and patients is a therefore a possible challenge. Some trial supervision will be provided to clinics by research staff, however the aim is to assess real-life implementation. Non-attendance by mothers for follow-up visits may lessen measured effectiveness of the intervention.

- Most patient records are stored in primary care for a maximum of 3 days, if at all. Both intervention and control clusters will be instructed to keep clinic records, which is a new concept, and may present challenges.

- Providers may not implement the intervention according to the trial protocol, for example if there is insufficient time during the clinic visit. This may reduce the effectiveness of the intervention, and will be explored in the process evaluation.

- Mothers in the intervention arm will be more familiar with child development activities than those in the control arm, which may bias recall of developmental achievements at 12 months. On-site observations of development will to try to reduce this bias.

\section{Comparison with existing literature}

To the authors' knowledge this study is the first to assess an early child development intervention, delivered in private GP clinics in Pakistan.

\section{Implications for practice}

Private sector engagement in education and healthcare delivery is a national strategy for universal coverage of health and social services in Pakistan, and the proposed intervention offers an alternative to a home-based delivery model of early child development care. If effective, service provision at private clinics may offer a more feasible option for populations in poor urban settings, where they are by far the most accessible provider of health care.

Trial registration

The trial has been registered with the Current Controlled Trials ISRCTN48032200.

\section{Funding}

This project is funded by Grand Challenge Canada, Saving Brains, and is implemented by the Association for Social Development, Pakistan (Ref. No. 0585-03). The views expressed and the information contained in this article are not necessarily endorsed by Grand Challenge Canada, Saving Brains.

Ethical approval

The trial has ethical approval from the National Bioethics Committee (NBC) Pakistan (Ref No NBC 154). The University of Leeds SoMREC approves technical support by University of Leeds staff (MREC15-094). National Bioethics Committee (NBC) Pakistan has granted ethical approval for the proposed project (Ref No NBC 154). Informed consent will be taken both at cluster and individual level.

Provenance

Freely submitted; externally peer reviewed.

\section{Acknowledgements}

We acknowledge the support of our partners, Institute of Child Health and The Children Hospital, The Institute of Psychiatry and Directorate General of Health Services Punjab, Pakistan for their technical guidance. 


\section{References}

1. Grantham-McGregor $S$, Cheung YB, Cueto $S$, et al. Developmental potential in the first 5 years for children in developing countries. Lancet 2007; 369(9555): 60-70. doi: 10.1016/S0140-6736(07)60032-4

2. Durkin MS, Hasan ZM, Hasan KZ. The ten questions screen for childhood disabilities: its uses and limitations in Pakistan. Journal of Epidemiology \& Community Health 1995; 49(4): 431-436. doi: 10.1136/jech.49.4.431

3. Yaqoob M, Ferngren H, Jalil F, et al. Early child health in Lahore, Pakistan: XII. milestones. Acta Paediatr 1993; 82(s391): 151-157. doi: 10.1111/j.1651-2227.1993.tb12913.x

4. Walker SP, Wachs TD, Gardner JM, et al. Child development: risk factors for adverse outcomes in developing countries. Lancet 2007; 369(9556): 145-157. doi: 10.1016/S0140-6736(07)60076-2

5. Avan BI, Raza SA, Kirkwood BR. An epidemiological study of urban and rural children in Pakistan: examining the relationship between delayed psychomotor development, low birth weight and postnatal growth failure. Trans R Soc Trop Med Hyg 2015; 109(3): 189-196. doi: 10.1093/trstmh/tru162

6. No Title. State of the World's Children statistical tables. http://www.unicef.org/infobycountry/pakistan pakistan_statistics.html. Published 2015.

7. Tough SC, Siever JE, Leew S, et al. Maternal mental health predicts risk of developmental problems at 3 years of age: follow up of a community based trial. BMC Pregnancy Childbirth 2008; 8:16. doi: 10.1186/ 1471-2393-8-16

8. Rahman A, lqbal Z, Harrington R. Life events, social support and depression in childbirth: perspectives from a rural community in the developing world. Psychol Med 2003; 33(7): 1161-1167. doi: 10.1017/ S0033291703008286

9. Gertler P, Heckman J, Pinto R, et al. Labor market returns to an early childhood stimulation intervention in Jamaica. Science 2014; 344(6187): 998-1001. doi: 10.1126/science.1251178

10. Walker SP, Chang SM, Vera-Hernández M, et al. Early childhood stimulation benefits adult competence and reduces violent behavior. Pediatrics 2011; 127(5). doi: 10.1542/peds.2010-2231

11. Kieling $\mathrm{C}$, Baker-Henningham $\mathrm{H}$, Belfer $\mathrm{M}$, et al. Child and adolescent mental health worldwide: evidence for action. Lancet 2011; 378(9801): 1515-1525. doi: 10.1016/S0140-6736(11)60827-1

12. Chang SM, Grantham-McGregor SM, Powell CA, et al. Integrating a parenting intervention with routine primary health care: a cluster randomized trial. Pediatrics 2015; 136(2): 272-280. doi: 10.1542/peds.20150119

13. Yousafzai AK, Rasheed MA, Rizvi A, et al. Effect of integrated responsive stimulation and nutrition interventions in the Lady Health Worker programme in Pakistan on child development, growth, and health outcomes: a cluster-randomised factorial effectiveness trial. Lancet 2014; 384(9950): 1282-1293. doi: 10. 1016/S0140-6736(14)60455-4

14. Khan SE, Khan MA, Owais SS, et al. Formative research for delivering early child development care at private clinics in poor urban localities of Punjab, Pakistan. Sci Int 2016; 28(3): 3131-3134.

15. Government of Pakistan. Pakistan bureau of statistics (Lahore). http://www.pbs.gov.pk/sites/default/files// pslm/publications/PSLM_2014-15_National-Provincial-District_report.pdf.

16. Government of Pakistan. Paksitan bureau of statistics (Rawalpindi). http://www.pbs.gov.pk/content/districtglance-rawalpindi.

17. Unicef. Integrating Early Childhood Development (ECD) activities into NutritionProgrammes in Emergencies. Why, What and How.... ECD Act into NutrProgram..... 2012. http://scholar.google.com/scholar?hl= en\&btnG $=$ Search\&q=intitle:Integrating+Early+Childhood+Development+(ECD)+activities+into+Nutrition + Programmes+in+Emergencies+.+Why+,+What+and+How\#0.

18. Twombly E, Fink G. Ages \& stages learning activities. 2004. http://asqoregon.com/asq/LearningActivities/ Learning Activities.pdf.

19. Mitchell AJ, Yadegarfar M, Gill J, et al. Case finding and screening clinical utility of the Patient Health Questionnaire (PHQ-9 and PHQ-2) for depression in primary care: a diagnostic meta-analysis of 40 studies. Br J Psychiatry Open 2016; 2(2): 127-138. doi: 10.1192/bjpo.bp.115.001685

20. Kroenke K, Spitzer RL, Williams JB. The patient health questionnaire-2: validity of a two-item depression screener. Med Care 2003; 41(11): 1284-1292. doi: 10.1097/01.MLR.0000093487.78664.3C

21. Cane J, O'Connor D, Michie S. Validation of the theoretical domains framework for use in behaviour change and implementation research. Implement Sci 2012; 7(1):37. doi: 10.1186/1748-5908-7-37

22. ASQ-3. An Introduction to ASQ- $3^{\mathrm{TM}}$ Why Screen? To detect delays early To encourage parent involvement and education. 2014. http://agesandstages.com/wp-content/uploads/2015/02/Training-PPT-for-website-103-14.pdf.

23 ASQ-3 User Manual. http://agesandstages.com/.

24. Filgueiras A, Pires $P$, Maissonette $S$, et al. Psychometric properties of the Brazilian-adapted version of the Ages and Stages Questionnaire in public child daycare centers. Early Hum Dev 2013; 89(8): 561-576. doi: 10.1016/j.earlhumdev.2013.02.005

25. Kvestad I, Taneja S, Kumar T, et al. The assessment of developmental status using the Ages and Stages questionnaire-3 in nutritional research in north Indian young children. Nutr J 2013; 12:50. doi: 10.1186/ 1475-2891-12-50

26. Squires J, Twombly E, Bricker D, et al. Psychometric studies of ASQ-3: Excerpted from ASQ-3 User's Guide. 2009. 3-6. 
27. Steenis LJ, Verhoeven $M$, Hessen DJ, et al. Parental and professional assessment of early child development: the ASQ-3 and the Bayley-III-NL. Early Hum Dev 2015; 91(3): 217-225. doi: 10.1016/j.earlhumdev.2015.01. 008

28. Vameghi R, Sajedi F, Kraskian Mojembari A, et al. Cross-cultural adaptation, validation and standardization of ages and stages questionnaire (ASQ) in iranian children. Iran J Public Health 2013; 42(5): 522-528.

29. Schonhaut L, Armijo I, Schönstedt $M$, et al. Validity of the ages and stages questionnaires in term and preterm infants. Pediatrics 2013; 131(5): e1468-e1474. doi: 10.1542/peds.2012-3313

30. Charafeddine L, Sinno D, Ammous F, et al. Adaptation to an Arabic speaking population and cultural sensitivity. Eur J Paediatr Neurol 2013; 17(5): 471-478. doi: 10.1016/j.ejpn.2013.03.001

31. ASQ-3. Concurrent Validity for ASQ-3 Questionnaire Intervals Concurrent Validity for ASQ-3 Questionnaire Intervals (continued). 2009.

32. Juneja M, Mohanty M, Jain R, et al. Ages and Stages Questionnaire as a Screening Tool for Developmental Delay in Indian Children. 2012.

33. IAPT. PHQ-9 Urdu. http://iapt.nhs.uk/silo/files/the-phq9-and-gad7-clinical-assessment-tools-in-urdu.pdf.

34. Galbraith S, Daniel JA, Vissel B. A study of clustered data and approaches to its analysis. J Neurosci 2010; 30(32): 10601-10608. doi: 10.1523/JNEUROSCI.0362-10.2010

35. Reed JF. Adjusted $\chi^{2}$ statistics: application to clustered binary data in primary care. Ann Fam Med 2004; 2 (3): 201-203. doi: 10.1370/afm.41

36. aod. Analysis of Overdispersed Data. https://cran.r-project.org/web/packages/aod/index.html. Accessed August 1, 2016.

37 Package GEE. ftp://cran.r-project.org/pub/R/web/packages/gee/gee.pdf. Accessed August 1, 2016.

38. Broström G, Holmberg H. Generalized linear models with clustered data: Fixed and random effects models. Comput Stat Data Anal 2011; 55(12): 3123-3134. doi: 10.1016/j.csda.2011.06.011

39. Slykerman RF, Thompson JM, Clark PM, et al. Determinants of developmental delay in infants aged 12 months. Paediatr Perinat Epidemiol 2007; 21(2): 121-128. doi: 10.1111/j.1365-3016.2007.00796.x

40. SPIRIT 2013. Evid Based Dent 2013; 14(4): 120. doi: 10.1038/sj.ebd.6400973

41. Moore G, Audrey S, Barker M, et al. Process evaluation of complex interventions. DecipherUkNet 2014; 1945: 64-75. doi:http://www.populationhealthsciences.org/MRC-PHSRN-Process-evaluation-guidance-final-2-. pdf.

42. Gale NK, Heath G, Cameron E, et al. Using the framework method for the analysis of qualitative data in multi-disciplinary health research. BMC Med Res Methodol 2013; 13(1):117. doi: 10.1186/1471-2288-13117 